\title{
Modified geometry of spur gear drives for compensation of shaft deflections
}

\author{
Ignacio Gonzalez-Perez • Victor Roda-Casanova • Alfonso Fuentes
}

Received: date / Accepted: date

\begin{abstract}
One of the greatest concern of spur gears is the edge contact of tooth surfaces that is caused by misalignment of the gear drive. Such a misalignment is caused partially by the deflections of the shafts where the gears are mounted. As a result of the edge contact a non favorable condition of bearing contact is achieved, providing a high level of contact and bending stresses. An intensive research and many practical solutions have been directed to modify the gear tooth surfaces in order to avoid edge contact. An innovative procedure is proposed here for: (i) determination of the errors of alignment at the gear drive caused by shaft deflections, (ii) incorporation of such errors of alignment in the generation process of spur gears for compensation of shaft deflections, and (iii), determination of a favorable function of transmission errors for the design load. A finite element model of a spur gear drive including pinion and wheel shafts is used for the determination of the errors of alignment along the cycle of meshing. Compensation of misalignments in gear generation is then accomplished by modification of pinion tooth surfaces whereas the wheel tooth surfaces are kept unmodified. Additional modifications of pinion tooth surfaces may
\end{abstract}

Ignacio Gonzalez-Perez

Polytechnic Universtiy of Cartagena

Department of Mechanical Engineering

Campus Muralla, Dr. Fleming s/n, 30202 Cartagena

Murcia, Spain

Tel.: +34-968326429

Fax: +34-968326449

E-mail: ignacio.gonzalez@upct.es

Victor Roda-Casanova

Universitat Jaume I, Castellon, Spain

Alfonso Fuentes

Polytechnic University of Cartagena, Spain be required for the obtention of a favorable function of transmission errors. The effect of several misalignment compensations in the reduction of contact stresses has been investigated. Postprocessing of load intensity functions and loaded transmission errors is included. The developed approach is illustrated with numerical examples.

Keywords gear geometry · tooth contact analysis · finite element analysis - loaded transmission errors . shaft deflections

\section{Introduction}

Spur gear drives are widely applied in the industry and have been a focus of intensive research to improve the load capacity and the conditions of meshing of involute profiles. First modifications on involute profiles, proposed in [1], were directed to remove some material from the borders of the tooth surfaces in order to minimize transmission errors for the design load. Many contributions in this area have been done $[2,3,4,5,6]$ looking for gear drives with reduced noise and vibration. Substitution of involute profile by other types of profiles has been another topic of intensive research. A pair of circular profiles for the generating cutters was proposed in [7] and mismatch of the circular profiles of the cutters was proposed for localization of the bearing contact in [8]. Modification of pinion tooth surfaces by application of parabolic profiles instead of straight profiles of the cutters has been proposed in [9] for the localization of the bearing contact. Localization of the bearing contact by the application of mismatched profiles of the cutters makes the contact less sensitive to the errors of alignment but, at the same time, increase contact and bending stresses, and transmission errors. 
A double crowned pinion tooth surface has been proposed in [10] for the localization of the bearing contact and the predesign of a parabolic function of unloaded transmission errors that control the transfer of meshing between adjacent pairs of teeth. A compromise solution was found in [11] combining a straight profile for the main part of the cutter tooth surface surrounded by parabolic profiles at the borders of the cutter tooth surfaces. This partial crowning keeps an involute area with no modification and allows: (i) reduction of the sensitivity of the bearing contact when errors of alignment are present, (ii) reduction of contact and bending stresses respect to a whole crowned tooth surface or respect to an involute tooth surface when errors of alignment are present, and (iii) reduction of unloaded transmission errors when misalignments are lower.

The determination of the appropriate topology of the gear tooth surfaces may not have a only solution. One of the main problems that may cost the shift of the bearing contact on the gear tooth surfaces is the deflection of the shafts where the gears are mounted, specially when the gears are installed out of the middle location between bearings. The deflection of the shafts implies then a misalignment of the wheel respect to the pinion and, consequently, the shift of the bearing contact. The main goal of this paper is to consider the shaft deflections, due to the design load, in the determination of the pinion tooth surface that provides, under misalignment, an almost conjugate action with the wheel tooth surface. This idea will allow to get a uniform distributed bearing contact for the design load. However, the bearing contact will be shifted when the load is far from the design load. Some additional modifications of the obtained pinion tooth surface may be required for the reduction of the sensitivity of the shift of the bearing contact and the reduction of transmission errors.

More specifically, the presented research has the following goals:

(1) Presentation of a method for determination of shaft deflections along the cycle of meshing between a pair of gears mounted on their corresponding shafts.

(2) Generation of the pinion tooth surface in misaligned conditions in order to have almost linear contact under the design load. The obtained geometry in misaligned condition is called compensated geometry.

(3) Determination of load intensity functions at every contact position of the cycle of meshing and comparison of such functions provided by the standard geometry, based on involute profiles, and the compensated geometry.

(4) Determination of loaded transmission errors along the cycle of meshing and modification of the compensated geometry to provide it with a predesign

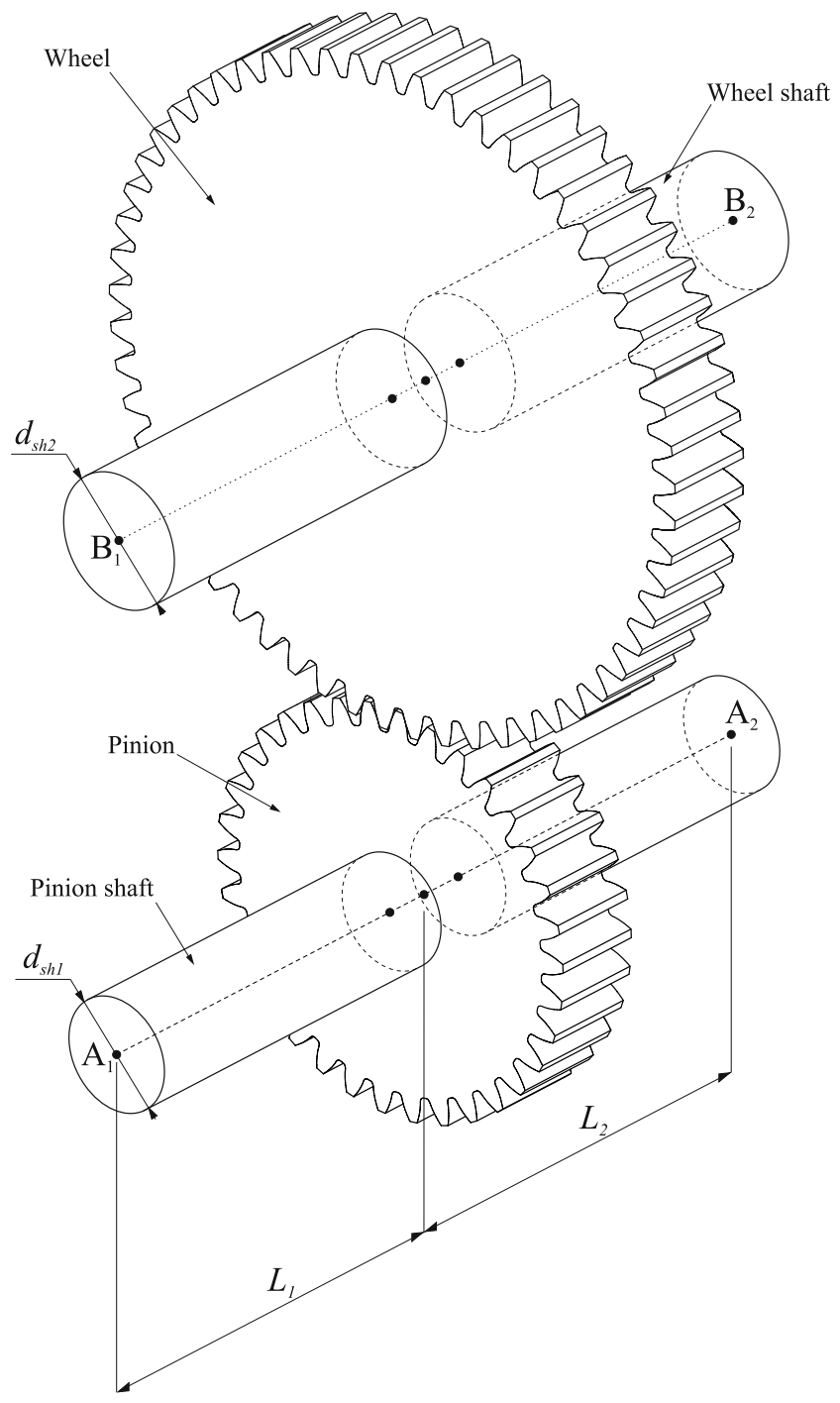

Fig. 1 Physical model of the gear drive

function of unloaded transmission errors. The predesign function of unloaded transmission errors will help reducing the resulting level of transmission errors.

The developed research is illustrated with numerical examples and has been applied to the physical model shown in Fig. 1. The physical model is based on two gears mounted in their corresponding shafts. The gears may be mounted out of the middle location between bearings by modifying lengths $L_{1}$ and $L_{2}$. This means that shaft deflections due to the design load will cost misalignment between pinion and wheel. A finite element model of the physical model shown in Fig. 1 has been built considering the ideas proposed in [12] for the development of the presented research. 


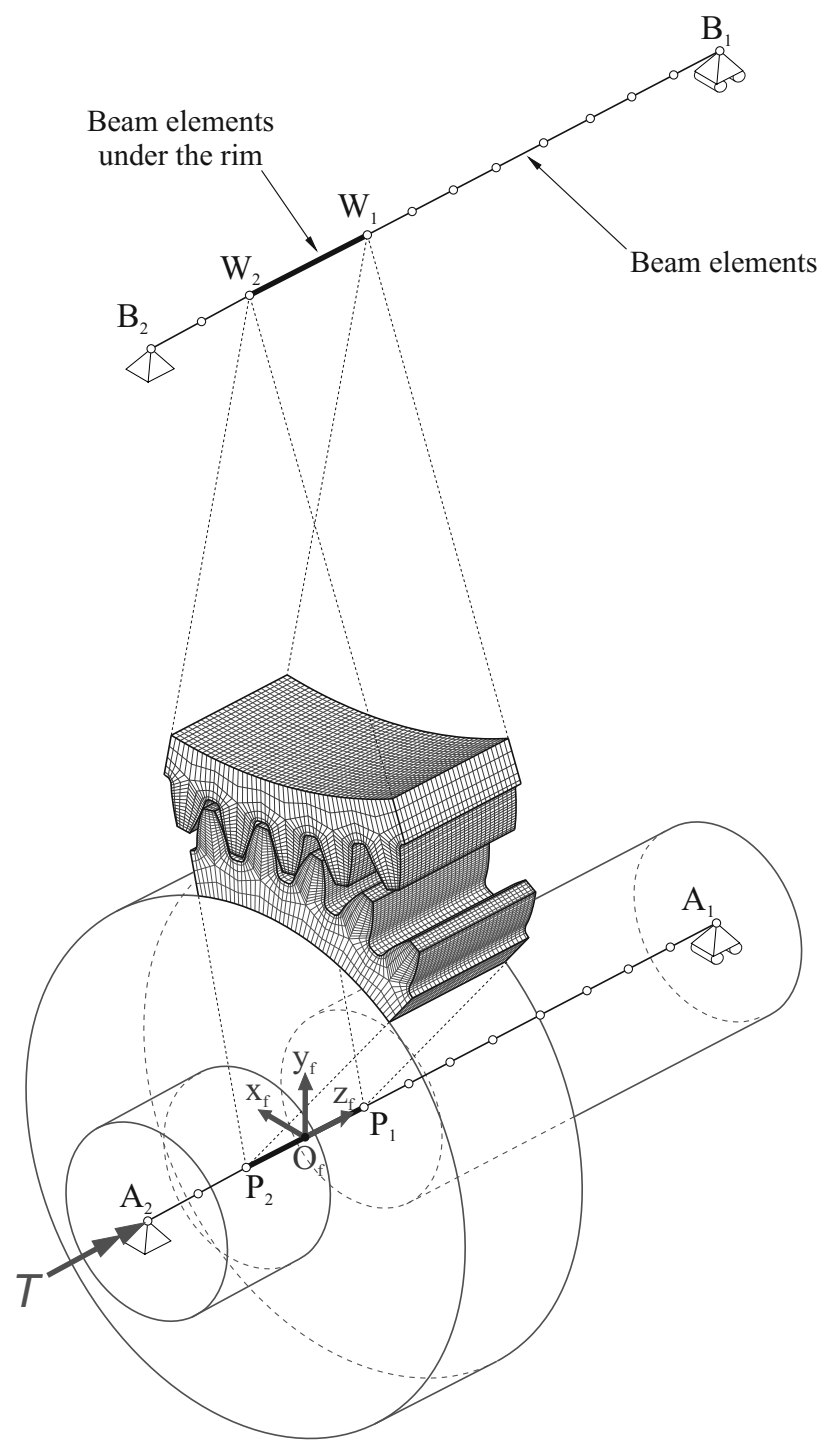

Fig. 2 Scheme of the finite element model

\section{Determination of gear misalignments due to shaft deflections}

Figure 2 shows schematically the finite element model of the physical model shown in Figure 1. Linear beam elements are considered for the modeling of shaft deflections. The transversal area of the shafts is considered as a property of the beam elements. For the portions of the shafts located under the pinion and wheel rims, the transversal area of the beam elements covers up to the bottom part of the rims. Torsional deformation is considered by modeling of rigid edges on the rim rigidly connected to the nodes of the beam elements that are located under the rim. A design load is applied by means of a torque $T$ at bearing $A_{2}$ while the rotation of the wheel shaft is blocked at node $B_{2}$. More

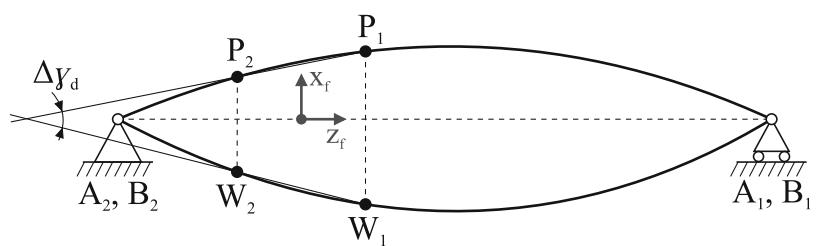

(a)

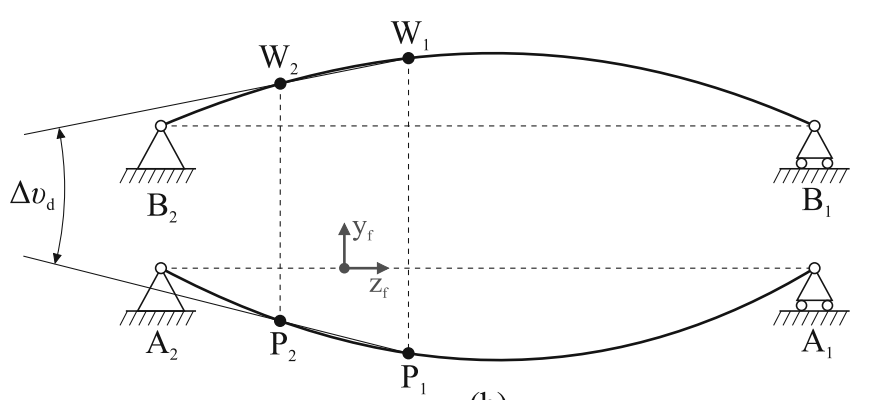

(b)

Fig. 3 For determination of misalignments $\Delta \gamma_{d}$ and $\Delta \nu_{d}$ due to shaft deflections

details of the schematic model shown in Figure 2 can be found in [12].

The misalignment between pinion and wheel is determined from the displacements that reference nodes $P 1, P 2, W 1$, and $W 2$ (see Fig. 2) experiment for the design load $T$ at each given contact position. The whole cycle of meshing is considered though a certain number of contact positions. The displacements $\left(u_{x i}, u_{y i}, u_{z i}\right)$ are obtained from the results of the finite element analysis for each contact position $i$ and are represented in the fixed coordinate system $S_{f}$, shown in Fig. 2. Mean values $\left(\bar{u}_{x}, \bar{u}_{y}, \bar{u}_{z}\right)$ are then determined at each reference node for the whole cycle of meshing.

The misalignment due to the tangential loading between the gear tooth surfaces is considered through the magnitude $\Delta \gamma_{d}$, shown in Fig. 3(a). Here, subindex $d$ means deflection, since shaft deflection costs the misalignment. Such a magnitude is determined as

$\Delta \gamma_{d}=\arctan \left[\frac{\bar{u}_{x}^{(W 1)}-\bar{u}_{x}^{(W 2)}}{b_{w}}\right]-\arctan \left[\frac{\bar{u}_{x}^{(P 1)}-\bar{u}_{x}^{(P 2)}}{b_{p}}\right](1)$

where $b_{p}$ and $b_{w}$ are the pinion and wheel face widths, respectively.

The misalignment due to the radial loading between the gear tooth surfaces is considered through the magnitude $\Delta \nu_{d}$, shown in Fig. 3(b). Such a magnitude is determined as

$\Delta \nu_{d}=\arctan \left[\frac{\bar{u}_{y}^{(W 1)}-\bar{u}_{y}^{(W 2)}}{b_{w}}\right]-\arctan \left[\frac{\bar{u}_{y}^{(P 1)}-\bar{u}_{y}^{(P 2)}}{b_{p}}\right]$

The relative displacements of the wheel respect to the pinion are considered through magnitudes $\Delta x_{d}, \Delta y_{d}$, 


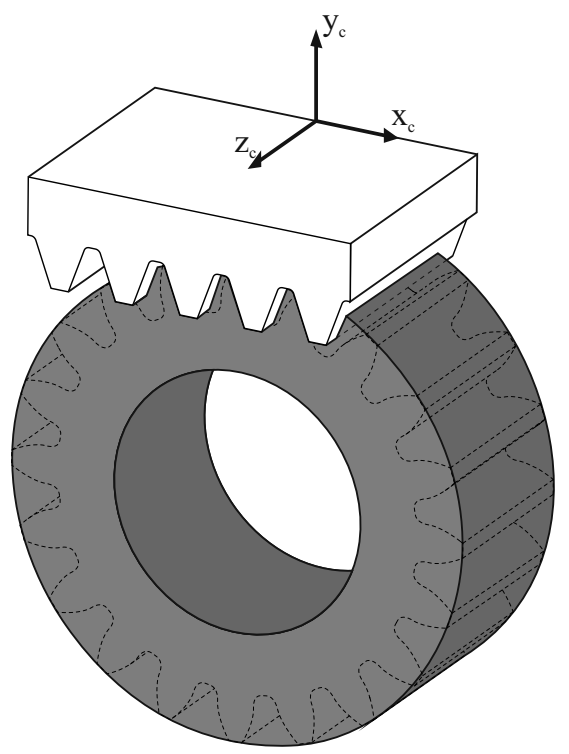

(a)

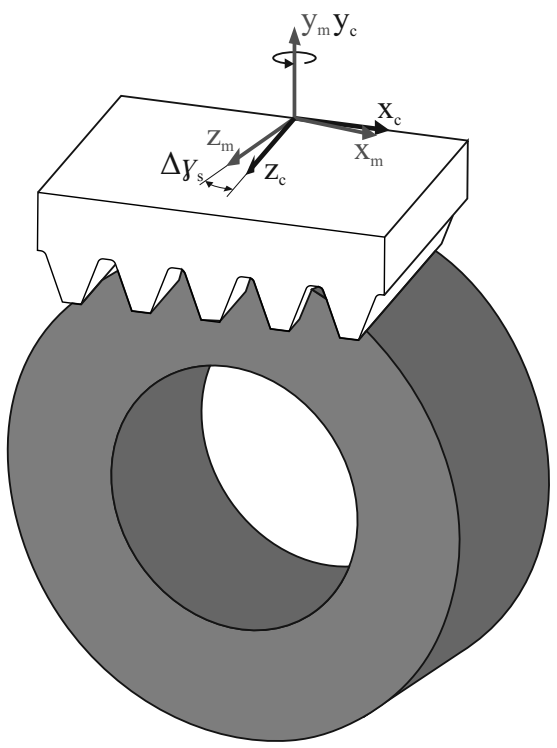

(b)

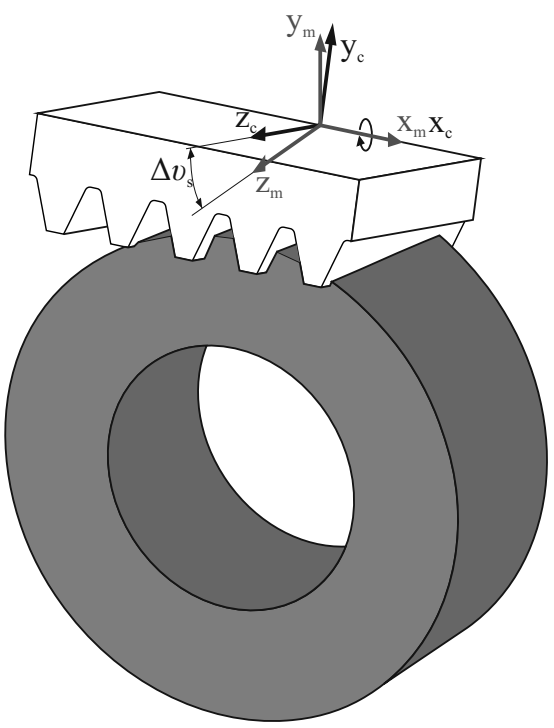

(c)

Fig. 4 Installment of the rack-cutter in: (a) a standard setting, (b) a setting with $\Delta \gamma_{s}$, and (c) a setting with $\Delta \nu_{s}$

and $\Delta z_{d}$, and are obtained as

$$
\begin{aligned}
& \Delta x_{d}=\left[\frac{\bar{u}_{x}^{(W 1)}+\bar{u}_{x}^{(W 2)}}{2}\right]-\left[\frac{\bar{u}_{x}^{(P 1)}+\bar{u}_{x}^{(P 2)}}{2}\right] \\
& \Delta y_{d}=\left[\frac{\bar{u}_{y}^{(W 1)}+\bar{u}_{y}^{(W 2)}}{2}\right]-\left[\frac{\bar{u}_{y}^{(P 1)}+\bar{u}_{y}^{(P 2)}}{2}\right] \\
& \Delta z_{d}=\left[\frac{\bar{u}_{z}^{(W 1)}+\bar{u}_{z}^{(W 2)}}{2}\right]-\left[\frac{\bar{u}_{z}^{(P 1)}+\bar{u}_{z}^{(P 2)}}{2}\right]
\end{aligned}
$$

\section{Gear generation}

Gear tooth surfaces can be analytically determined from any cutter regular tooth surface following the modern theory of gearing [13]. Two coordinate systems $S_{c}$ and $S_{1}$ are considered rigidly connected to the cutter and the pinion tooth surfaces. Coordinate transformation from system $S_{c}$ to system $S_{1}$ and observation of the equation of meshing allow the pinion tooth surface to be determined from the rack-cutter tooth surface

$\mathbf{r}_{1}(u, v, \psi)=\mathbf{M}_{1 c}\left(\psi_{1}\right) \mathbf{r}_{c}(u, v)$

$\left(\frac{\partial \mathbf{r}_{1}}{\partial u} \times \frac{\partial \mathbf{r}_{1}}{\partial v}\right) \cdot \frac{\partial \mathbf{r}_{1}}{\partial \psi}=0$

Here, $(u, v)$ are the cutter surface parameters and $\psi$ is the generalized parameter of generation. Matrix $\mathbf{M}_{1 c}$ allows coordinate transformation from system $S_{c}$ to system $S_{1}$. Simultaneous consideration of equations 6 and 7 allows pinion tooth surface to be determined.

Figure 4(a) shows the standard setting of a rackcutter over the pinion being generated. Settings of the rack-cutter with angular magnitudes $\Delta \gamma_{s}$ and $\Delta \nu_{s}$ are shown in Figures 4(b) and 4(c), respectively. Here, coordinate system $S_{m}$ is an auxiliary fixed coordinate system that is parallel to system $S_{f}$ (see Fig.2). System $S_{c}^{\prime}$ is an auxiliary coordinate system parallel to system $S_{c}$, not shown in Fig. 4. The rack-cutter can also be set with some displacement values $\Delta x_{s}, \Delta y_{s}$, and $\Delta z_{s}$ respect to the standard setting. As a result of the setting of the cutter with some of those magnitudes mentioned above, a modified pinion tooth surface may be obtained.

The purpose of generation of the pinion with a misaligned cutter is to be able to obtain a pinion tooth surface that will be conjugated to the wheel tooth surface when shaft deflections due to the design load are present. The misalignment of the wheel respect to the pinion is caused partially by shaft deflections. Magnitudes $\Delta \gamma_{d}, \Delta \nu_{d}, \Delta x_{d}, \Delta y_{d}$, and $\Delta z_{d}$, are obtained for the design load as it has been explained in previous section. These misalignment values will be considered as the settings for the generation of the pinion by its rack-cutter. In this way, the teeth of rack-cutter are in the same relative location respect to the pinion as the teeth of the misaligned wheel.

$\Delta \gamma_{s}=\Delta \gamma_{d}$

$\Delta \nu_{s}=\Delta \nu_{d}$

$\Delta x_{s}=\Delta x_{d}$

$\Delta y_{s}=\Delta y_{d}$

$\Delta z_{s}=\Delta z_{d}$

Additionally, the pinion tooth surface can be generated by cutter installed with misalignments and provided with modified profiles. In such a case, the pinion 


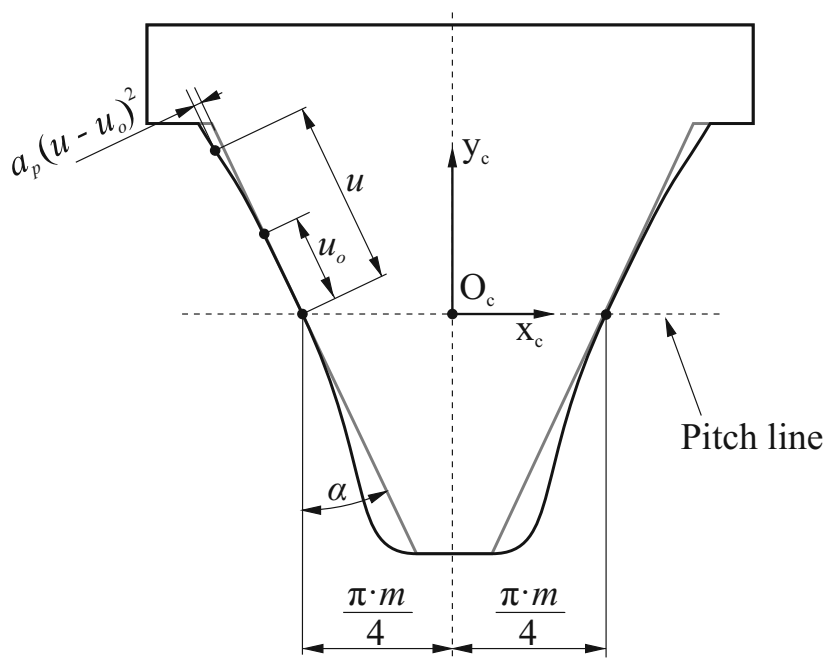

(a)

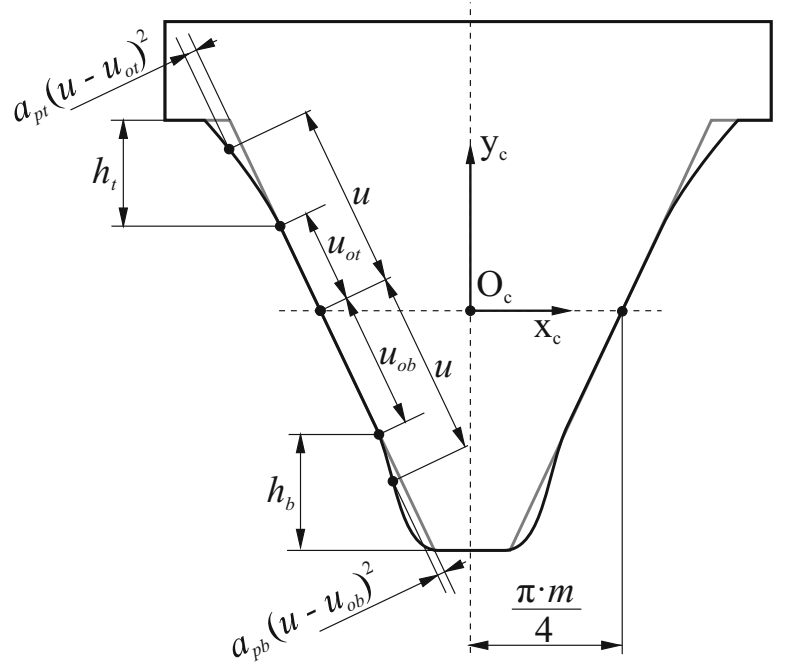

(b)

Fig. 5 Definition of the modified profiles of a rack-cutter for the application of (a) parabolic profile crowning and (b) parabolic relieves at top and bottom sides

tooth surfaces are modified by two actions, (i) the misaligned installment of the cutter, and (ii) the modified profiles of the cutter. Different types of profiles can be applied instead of the standard straight profile. Figure 5 shows two examples of definition of modified profiles in the normal section of a rack-cutter. Figure 5(a) shows the definition of a parabolic profile as the main active profile. A parabola is defined by the parabola coefficient $a_{p}$ and the profile parameter $u_{o}$ for the location of the parabola apex respect to the pitch line. Profile parameter $u$ is measured along the straight reference profile whereas longitudinal parameter $v$ is not shown in Fig. 5(a). Figure 5(b) shows another type of modified profile based on a straight profile and parabolic relieves at top and bottom sides. Parabola coefficients $a_{p b}$ and $a_{p t}$ are considered for definition of the parabolas at top and bottom sides, respectively. Parabola apexes are located by magnitudes $u_{o t}$ and $u_{o b}$ or, alternatively, by distances $h_{t}$ and $h_{b}$.

\section{Computerized simulation of gear meshing and determination of unloaded transmission errors}

A general purpose algorithm has been applied for computerized simulation of gear meshing between pinion and wheel. It is based on a numerical method that takes into account the position of the surfaces and minimize the distances until contact is achieved, based on the work [14] and applied later in the works [15, 16]. This algorithm assumes rigid body behavior of tooth surfaces and can be applied to the analysis of gear drives in point, lineal, or edge contact. In the present work, no user defined misalignments will be considered for gear meshing investigation. The relation between the angle of rotation of the pinion, $\phi^{(1)}$, and the angle of rotation of the wheel, $\phi^{(2)}$, will provide the function of unloaded transmission errors, which will depend on the geometries of pinion and wheel tooth surfaces.

Transmission error is considered as the angular difference between the actual position of the wheel and the theoretical position of the wheel respect to the pinion. It is considered positive when the wheel moves away from the pinion and negative when the wheel moves towards the pinion. The function of unloaded transmission errors is obtained as the discrete function

$\Delta \phi_{i}^{(u)}=\left(-\phi_{i}^{(2)}-\phi_{i}^{(1)} \frac{N_{1}}{N_{2}}\right)$

where $\phi_{i}^{(1)}$ and $\phi_{i}^{(2)}$ are the angular rotations that allows pinion and wheel, respectively, to become in contact under no load at each contact position $i$. Here, superscript (u) means unloaded, $N_{1}$ and $N_{2}$ represents the number of teeth of pinion and wheel, respectively. The minus sign before angle $\phi_{i}^{(2)}$ is required since wheel rotation is considered negative in clockwise direction and $\phi_{i}^{(2)}$ makes the wheel to move away from the pinion.

\section{Determination of load intensity functions and loaded transmission errors}

Load intensity functions are determined considering the pressure distribution over the tooth surfaces obtained from the finite element analysis for each contact position. Details of determination of load intensity functions are described in [12]. Figure 6 shows an example 




Fig. 6 Example of load intensity function along the face width for a bearing contact that is shifted

of a load intensity function when the bearing contact is shifted.

Loaded transmission errors are obtained for the whole cycle of meshing considering tooth bending deformations, contact deformations and torsional deformations of pinion and wheel. Torsional deformations of shafts are also considered. The procedure for the determination of the loaded transmission errors is as follows:

(i) Nodal rotations $\theta_{i}^{(P 1)}, \theta_{i}^{(P 2)}, \theta_{i}^{(W 1)}$, and $\theta_{i}^{(W 2)}$ at nodes $P 1, P 2, W 1, W 2$ are obtained at each contact position $i$ from the finite element analysis.

(ii) Mean values of pinion and wheel rotations for each contact position are then obtained as

$\bar{\theta}_{i}^{(p)}=\frac{\theta_{i}^{(P 1)}+\theta_{i}^{(P 2)}}{2}$

$\bar{\theta}_{i}^{(w)}=\frac{\theta_{i}^{(W 1)}+\theta_{i}^{(W 2)}}{2}$

where $\bar{\theta}_{i}^{(p)}$ results positive when pinion shaft rotates in counterclockwise direction and $\bar{\theta}_{i}^{(w)}$ results negative when wheel shaft rotates in clockwise direction.

(iii) Since the nodal rotation of node $B 2$ (see Fig. 2) is blocked, $-\bar{\theta}_{i}^{(w)}$ represents the wheel rotation due to torsional deformation of the wheel shaft between the end node $B 2$ and the wheel location. The minus sign is required to make it positive, since the torsional deformation of the wheel shaft makes the wheel to move away from the pinion.

(iv) Since the pinion shaft is free to rotate, $\bar{\theta}_{i}^{(p)}$ represents the pinion rotation due to tooth contact deformations, tooth bending deformations, and torsional deformations of pinion and wheel. To account for all these deformations in the determination of the transmission error, $-\bar{\theta}_{i}^{(p)} \cdot N_{1} / N_{2}$ will represent the rotation of the wheel towards the pinion due to deformations. The minus sign is required to make it negative, since the deformations make the wheel to move closer to the pinion.

(v) Finally, the loaded transmission error is obtained as a discrete function by

$\Delta \phi_{i}^{(l)}=\left(-\bar{\theta}_{i}^{(w)}-\bar{\theta}_{i}^{(p)} \frac{N_{1}}{N_{2}}\right)$
Here, superscript $l$ means loaded.

The torsional deformation of the pinion shaft between the end node $A 2$ (see Fig. 2), where the torque is applied, and the pinion location, is not included in the determination of the loaded transmission error between pinion and wheel.

The set of results $\Delta \phi_{i}^{(l)}$ represents the function of loaded transmission errors. Such a function has to be added to the function of unloaded transmission errors $\Delta \phi_{i}^{(u)}$ to obtain the total function of transmission errors

$\Delta \phi_{i}=\Delta \phi_{i}^{(u)}+\Delta \phi_{i}^{(l)}$

The peak-to-peak transmission error is then defined as

$\Delta \phi_{\max }=\max \left(\Delta \phi_{i}\right)-\min \left(\Delta \phi_{i}\right)$

The predesign of function $\Delta \phi_{i}^{(u)}$ may help reducing the value of $\Delta \phi_{\max }$. This means that the predesign of the function $\Delta \phi_{i}^{(u)}$ may partially compensate the function $\Delta \phi_{i}^{(l)}$.

\section{Numerical examples}

Table 1 shows the main design data of the spur gear drive shown in Fig. 1. The gear drive is misplaced from the middle location between bearings by distances $L_{1}$ and $L_{2}$. The pinion is rotated in counterclockwise direction by the action of an applied torque $T$ at bearing $A_{2}$ (see Fig. 2). A finite element model of 94211 elements and 121523 nodes has been considered.

Table 1 Design data of the spur gear drive represented in Fig. 1

\begin{tabular}{lr} 
Magnitudes & Values \\
\hline Module, $m[\mathrm{~mm}]$ & 3.0 \\
Pressure angle, $\alpha$ [degrees] & 20.0 \\
Tooth number of the pinion, $N_{1}$ & 34 \\
Tooth number of the wheel, $N_{2}$ & 57 \\
Face width, $b=b_{p}=b_{w}[\mathrm{~mm}]$ & 25.0 \\
Pinion shaft diameter, $d_{s h 1}[\mathrm{~mm}]$ & 30.0 \\
Wheel shaft diameter, $d_{s h 2}[\mathrm{~mm}]$ & 35.0 \\
Young's Modulus, $E[\mathrm{MPa}]$ & 206800 \\
Poisson's ratio, $\nu$ & 0.29 \\
Applied torque, $T[\mathrm{Nm}]$ & 290.0 \\
Distance bearing A2 - pinion, $L_{1}[\mathrm{~mm}]$ & 100.0 \\
Distance pinion - bearing $A 1, L_{2}[\mathrm{~mm}]$ & 40.0 \\
\hline
\end{tabular}

A total of $i=21$ contact positions are considered distributed along two cycles of meshing. In order to obtain shaft deflections, a standard geometry $\Sigma_{s}$ of the pinion and a design load of $T=290.0 \mathrm{Nm}$ are considered. For the determination of standard geometry $\Sigma_{s}$, a 




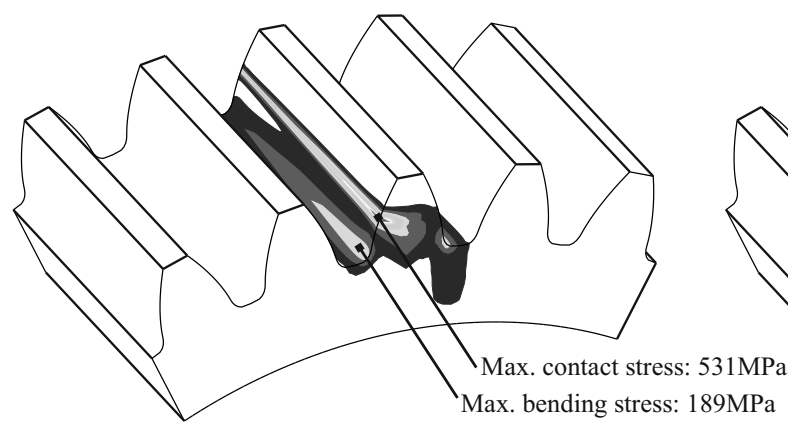

(a)

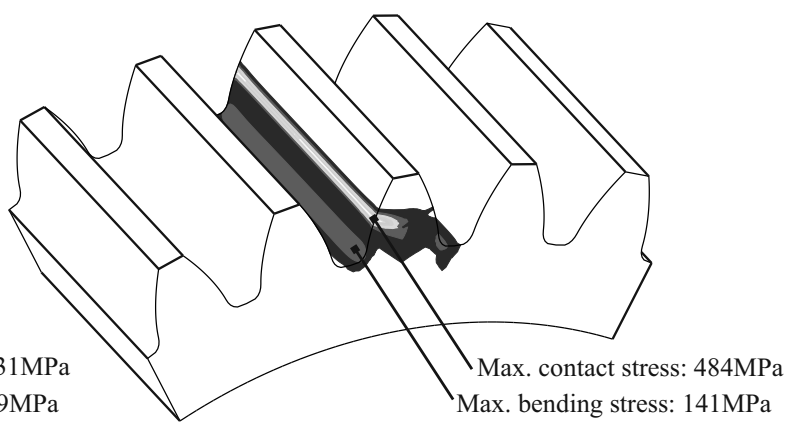

(b)

Fig. 7 Formation of the bearing contact and maximum contact and bending stresses at contact position 11 for (a) standard geometry $\Sigma_{s}$ and (b) compensated geometry $\Sigma_{c 1}$

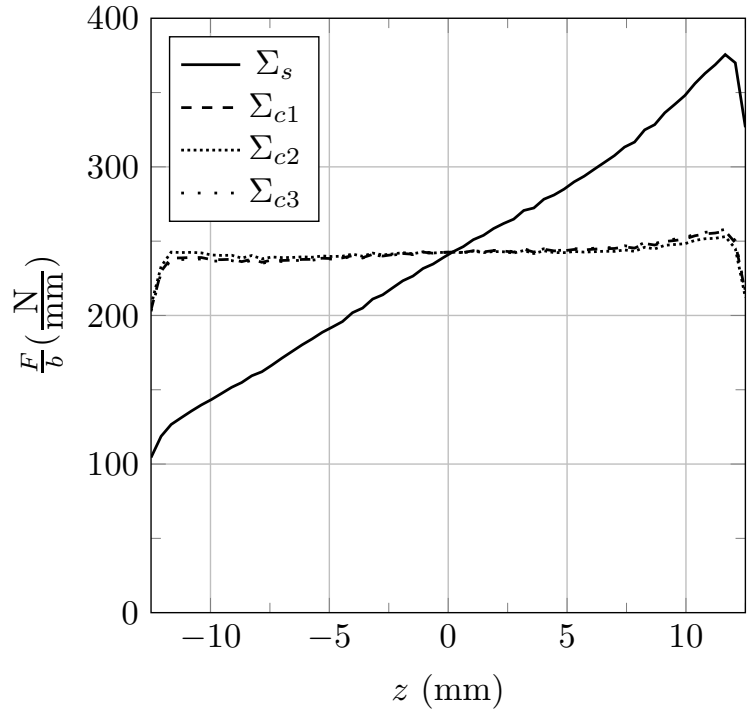

(a)

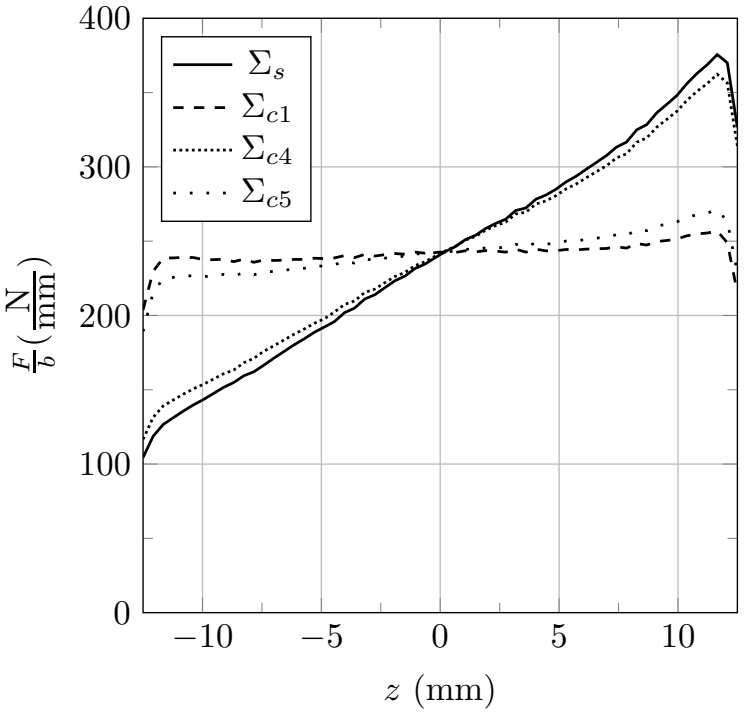

(b)

Fig. 8 Load intensity functions for standard geometry and compensated geometries at contact position 11: (a) comparison between $\Sigma_{s}$ and $\Sigma_{c 1}, \Sigma_{c 2}$, and $\Sigma_{c 3}$; (b) comparison between $\Sigma_{s}$ and $\Sigma_{c 1}, \Sigma_{c 4}$, and $\Sigma_{c 5}$

cutter with straight profiles installed in a standard setting (see Fig. 4(a)) is considered. Among the 21 contact positions, the maximum, minimum, and mean values of gear misalignments due to shaft deflections are obtained and shown in Table 2 .

Table 2 Gear misalignments due to shaft deflections obtained for the standard geometry $\Sigma_{s}$ and a torque of 290.0 $\mathrm{Nm}$

\begin{tabular}{lccc} 
& Max. values & Min. values & Mean values \\
\hline$\Delta \gamma_{d}[$ degrees $]$ & $-0,024266$ & $-0,023326$ & $-0,023551$ \\
$\Delta \nu_{d}[$ degrees $]$ & $-0,008822$ & $-0,008439$ & $-0,008546$ \\
$\Delta x_{d}[\mathrm{~mm}]$ & 0,026187 & 0,025650 & 0,025776 \\
$\Delta y_{d}[\mathrm{~mm}]$ & $-0,009536$ & $-0,009311$ & $-0,009378$ \\
$\Delta z_{d}[\mathrm{~mm}]$ & 0,0 & 0,0 & 0,0 \\
\hline
\end{tabular}

Several compensated geometries of the pinion are then obtained by considering the gear misalignments shown in Table 2 as the settings for the installment of the cutter (see Section 3). For the obtention of these geometries, the cutter is provided with straight profiles. Five types of compensated geometries have been considered:

- Geometry $\Sigma_{c 1}$ is generated considering the mean values of gear misalignments shown in Table 2 .

- Geometry $\Sigma_{c 2}$ is generated considering the maximum values of gear misalignments shown in Table 2 .

- Geometry $\Sigma_{c 3}$ is generated considering the minimum values of gear misalignments shown in Table 2.

- Geometry $\Sigma_{c 4}$ is generated considering just the mean value of $\Delta \nu_{d}$ shown in Table 2.

- Geometry $\Sigma_{c 5}$ is generated considering just the mean value of $\Delta \gamma_{d}$ shown in Table 2.

Figure 7 shows the formation of the bearing contact on the pinion tooth surfaces at contact position 11 for geometries $\Sigma_{s}$ and $\Sigma_{c 1}$ when a design load of $T=290.0$ 


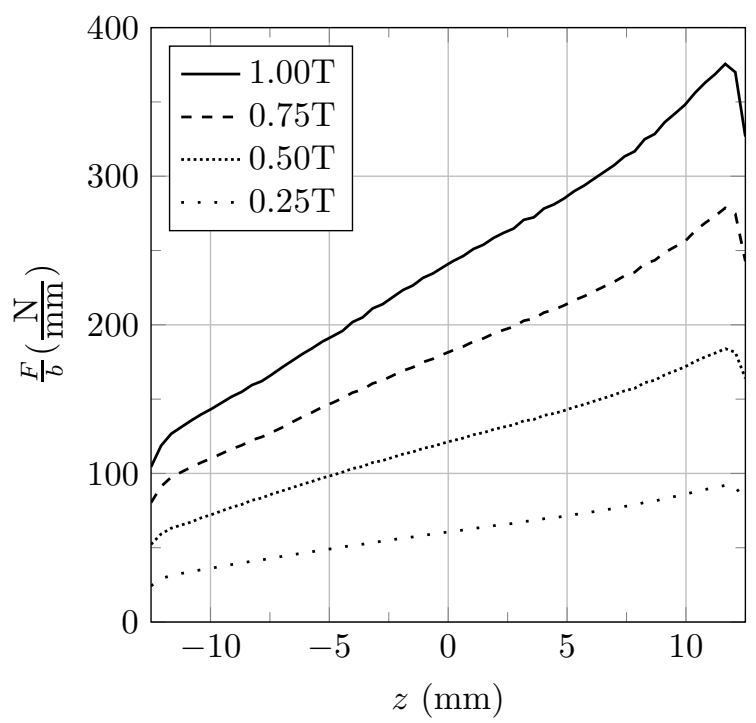

(a)

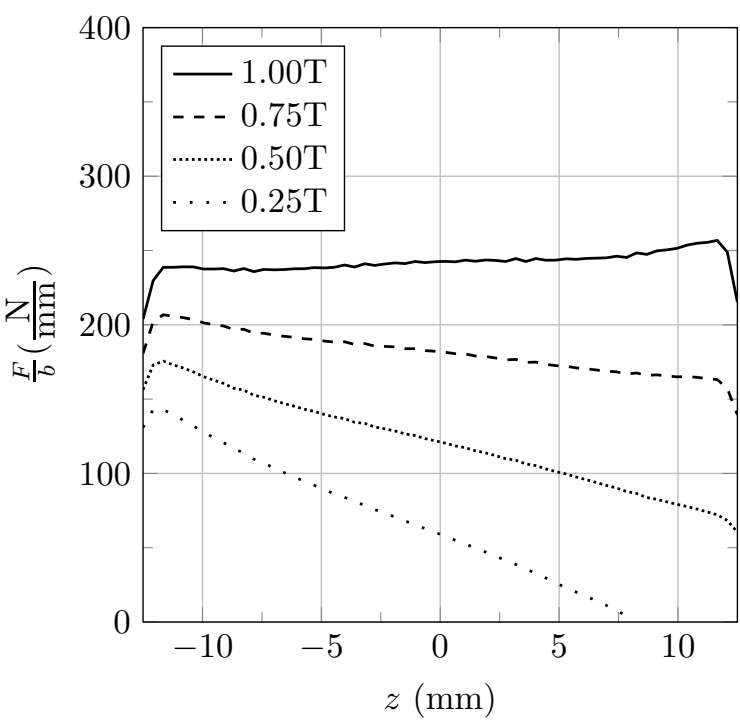

(b)

Fig. 9 Load intensity functions at contact position 11 for several values of applied torque $T=290.0 \mathrm{Nm}$ at (a) standard geometry $\Sigma_{s}$ and (b) compensated geometry $\Sigma_{c 1}$

$\mathrm{Nm}$ is applied. Figure 7(a) illustrates that the bearing contact is unevenly distributed and shifted towards the front face of the pinion when the standard geometry $\Sigma_{s}$ is considered. However, when the compensated geometry $\Sigma_{c 1}$ is considered (see Fig. $7(\mathrm{~b})$ ), the bearing contact is uniformly distributed, providing a reduction on Mises contact stress about 8.85 percent and a reduction on Mises bending stress about 26.5 percent.

The standard geometry and the compensated geometries mentioned above are compared through the load intensity functions obtained at contact position 11. Figure 8(a) shows the load intensity functions for geometries $\Sigma_{s}, \Sigma_{c 1}, \Sigma_{c 2}$, and $\Sigma_{c 3}$. The load intensity function provided by geometry $\Sigma_{s}$ shows an important increment of the load intensity due to shaft deflections. However, the function provided by geometry $\Sigma_{c 1}$ shows an uniform distribution of the load intensity. Figure $8($ a) shows as well that the load intensity functions provided by geometries $\Sigma_{c 1}, \Sigma_{c 2}$, and $\Sigma_{c 3}$ are very similar each other.

Figure 8(b) illustrates that the main contribution to get an uniform distribution of the load intensity and compensate the shaft deflections is due to magnitude $\Delta \gamma_{d}$, since the load intensity function provided by geometry $\Sigma_{c 5}$ is very close to the one provided by geometry $\Sigma_{c 1}$. However, the load intensity function provided by geometry $\Sigma_{c 4}$ is very close to the one provided by geometry $\Sigma_{s}$, showing a minor influence of magnitude $\Delta \nu_{d}$ for compensation of shaft deflections.

Regarding the behavior of geometries $\Sigma_{s}$ and $\Sigma_{c 1}$ when the load is different from the design load, Figure 9 illustrates the load intensity functions for both geome-

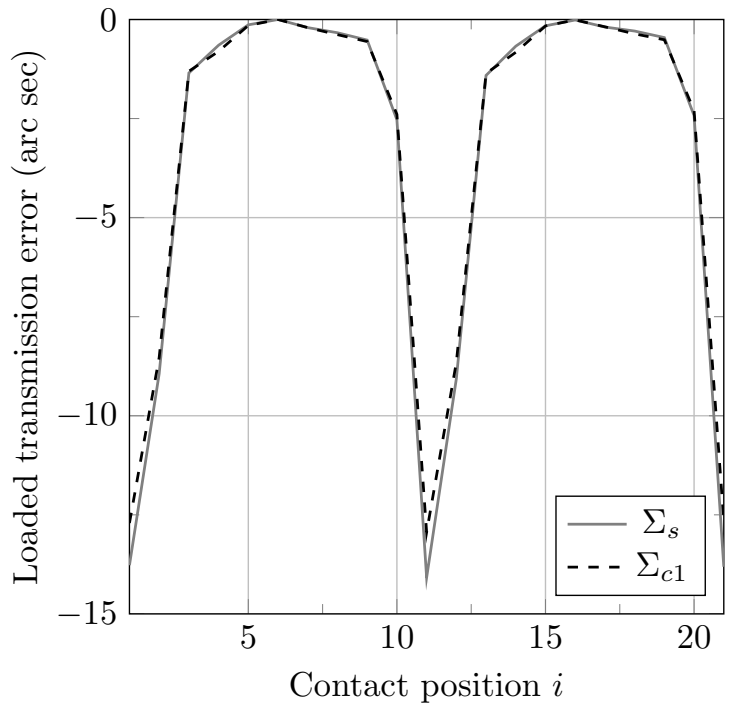

Fig. 10 Functions of loaded transmission errors for geometries $\Sigma_{s}$ and $\Sigma_{c 1}$

tries and different values of the applied torque from $0.1 T$ up to $1.0 T$. The results show that under a low load, the load intensity is more uniformly distributed on the standard geometry $\Sigma_{s}$ than on the compensated geometry $\Sigma_{c 1}$. However and as it is expected, the load intensity becomes more uniformly distributed when the load is increased up to the design load for the compensated geometry $\Sigma_{c 1}$.

Regarding gear meshing results (see section 4), standard geometry $\Sigma_{s}$ and compensated geometry $\Sigma_{c 1}$ provides a function of zero unloaded transmission errors due to the conjugated action between pinion and wheel 


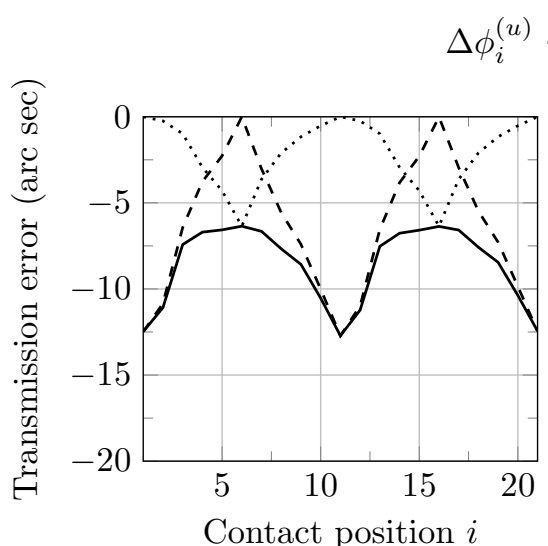

(a)

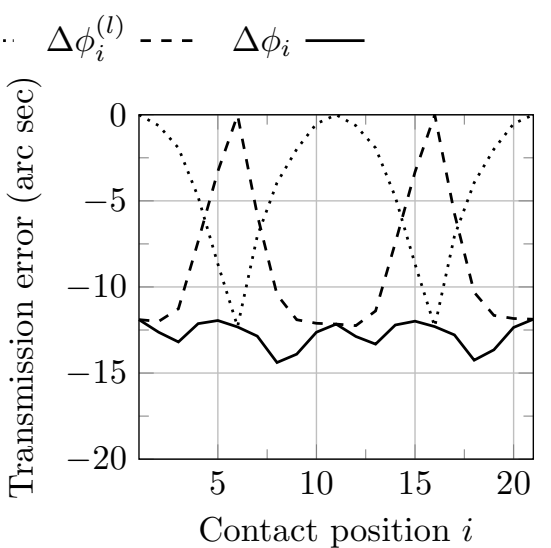

(b)

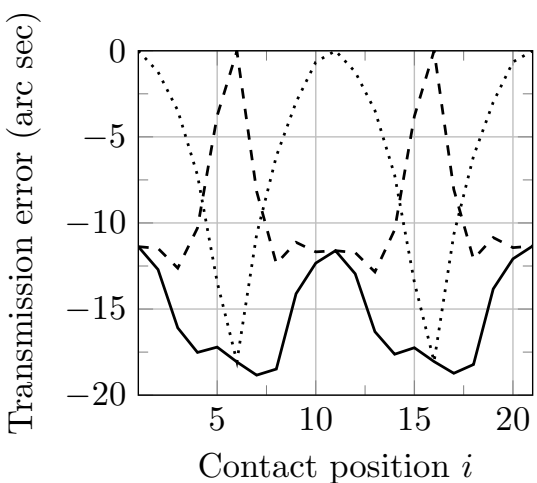

(c)

Fig. 11 Functions of transmission errors (unloaded, loaded, and total) for geometries: (a) $\Sigma_{c 1 m 1}$, (b) $\Sigma_{c 1 m 2}$, and (c) $\Sigma_{c 1 m 3}$

tooth surfaces when no load is applied. The function of transmission errors is then obtained as the function of loaded transmission errors (see section 5). Figure 10 shows the functions of loaded transmission errors for geometries $\Sigma_{s}$ and $\Sigma_{c 1}$. Two cycles of meshing are observed. The peak-to-peak value of transmission error is about 14.08 arcsec for geometry $\Sigma_{s}$ and 12.94 arcsec for geometry $\Sigma_{c 1}$.

Several compensated modified geometries $\Sigma_{c 1 m i}(i=$ $1, \ldots, 11)$ are generated for the investigation of the effect of the predesign of a function of unloaded transmission errors in the reduction of the peak-to-peak value of total transmission errors. Tables 3 and 4 show the main design parameters of the compensated modified geometries. Three compensated modified geometries are based on a whole crowning of the pinion tooth surfaces by application of a rack-cutter with parabolic profiles. Eight compensated modified geometries are based on a partial crowning of the pinion tooth surfaces by application of a rack-cutter with straight profile and parabolic relieves at bottom and top sides.

Table 3 Compensated modified geometries by application of whole crowning by parabolic profiles (see Fig. 5(a))

\begin{tabular}{ccc} 
Geometry & $a_{p}\left[\mathrm{~mm}^{-1}\right]$ & $u_{o}[\mathrm{~mm}]$ \\
\hline$\Sigma_{c 1 m 1}$ & 0,001 & $-0,4$ \\
$\Sigma_{c 1 m 2}$ & 0,002 & $-0,4$ \\
$\Sigma_{c 1 m 3}$ & 0,003 & $-0,4$ \\
\hline
\end{tabular}

Figure 11 shows the functions of transmission errors (unloaded, loaded, and total) for geometries $\Sigma_{c 1 m 1}$, $\Sigma_{c 1 m 2}$, and $\Sigma_{c 1 m 3}$. The lowest peak-to-peak value of total transmission errors is reached at geometry $\Sigma_{c 1 m 2}$, representing such a geometry the best solution from the three considered geometries.
Table 4 Compensated modified geometries by application of partial crowning by parabolic relieves (see Fig. 5(b))

\begin{tabular}{ccccc} 
Geometry & $a_{p t}\left[\mathrm{~mm}^{-1}\right]$ & $h_{t}[\mathrm{~mm}]$ & $a_{p b}\left[\mathrm{~mm}^{-1}\right]$ & $h_{b}[\mathrm{~mm}]$ \\
\hline$\Sigma_{c 1 m 4}$ & 0,005 & 2,5 & 0,005 & 2,5 \\
$\Sigma_{c 1 m 5}$ & 0,010 & 2,5 & 0,010 & 2,5 \\
$\Sigma_{c 1 m 6}$ & 0,015 & 2,5 & 0,015 & 2,5 \\
$\Sigma_{c 1 m 7}$ & 0,020 & 2,5 & 0,020 & 2,5 \\
$\Sigma_{c 1 m 8}$ & 0,004 & 3,0 & 0,004 & 3,0 \\
$\Sigma_{c 1 m 9}$ & 0,008 & 3,0 & 0,008 & 3,0 \\
$\Sigma_{c 1 m 10}$ & 0,012 & 3,0 & 0,012 & 3,0 \\
$\Sigma_{c 1 m 11}$ & 0,016 & 3,0 & 0,016 & 3,0 \\
\hline
\end{tabular}

Figure 12 shows the functions of transmission errors for geometries $\Sigma_{c 1 m 4}, \Sigma_{c 1 m 5}, \Sigma_{c 1 m 6}$, and $\Sigma_{c 1 m 7}$ where $h_{t}=h_{b}=2,5 \mathrm{~mm}$ (see Fig. 5(b)). Figure 12(a) illustrates the increment of the peak-to-peak value of unloaded transmission errors when parabola coefficient is increased, whereas Figure 12(b) shows that the peakto-peak value of loaded transmission errors is decreased due in part to the reduction of contact ratio. Figure 12(c) shows that the minimum peak-to-peak value of total transmission error is found for geometry $\Sigma_{c 1 m 5}$ from the four considered geometries.

Similar results can be found in Fig. 13 for compensated modified geometries $\Sigma_{c 1 m 8}, \Sigma_{c 1 m 9}, \Sigma_{c 1 m 10}$, and $\Sigma_{c 1 m 11}$ where $h_{t}=h_{b}=3,0 \mathrm{~mm}$. The minimum peakto-peak value of total transmission error is found for geometry $\Sigma_{c 1 m 9}$ from the four considered geometries.

Regarding stress analysis results, it is important to notice that the developed research has been perform along two cycles of meshing and not just at one contact position. Figure 14 shows the evolution of contact and bending stresses for geometries $\Sigma_{s}, \Sigma_{c 1}, \Sigma_{c 1 m 2}$, $\Sigma_{c 1 m 5}$, and $\Sigma_{c 1 m 9}$. It is observed, along the cycle of meshing, a reduction of contact and bending stresses in the compensated geometries respect to the standard ge- 




(a)

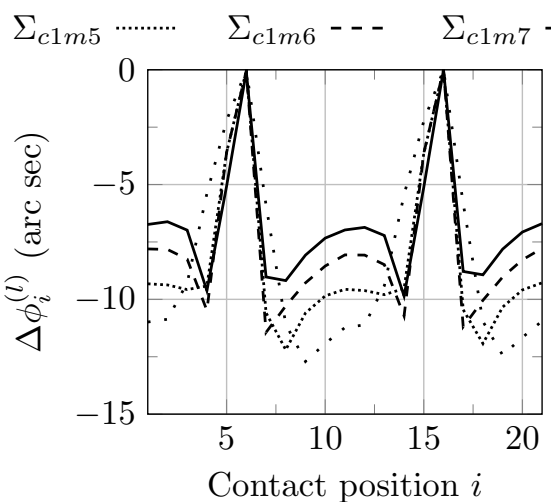

(b)

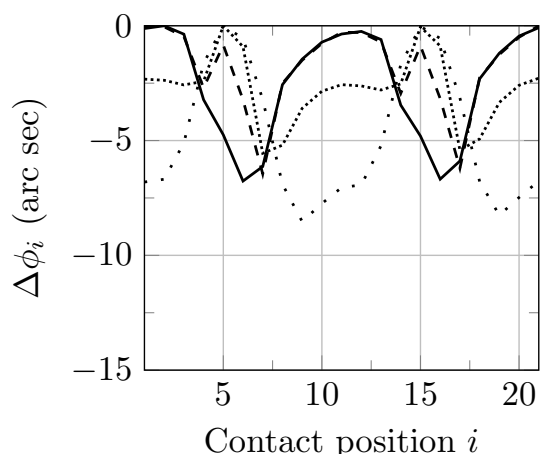

(c)

Fig. 12 Functions of (a) unloaded transmission errors, (b) loaded transmission errors, and (c) total transmission errors for geometries $\Sigma_{c 1 m 4}, \Sigma_{c 1 m 5}, \Sigma_{c 1 m 6}$, and $\Sigma_{c 1 m 7}$

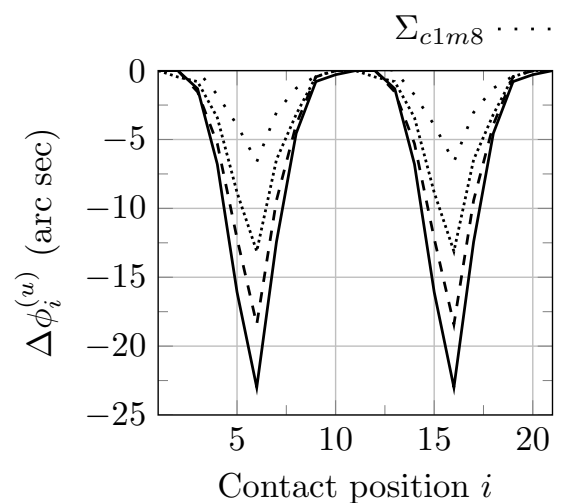

(a)

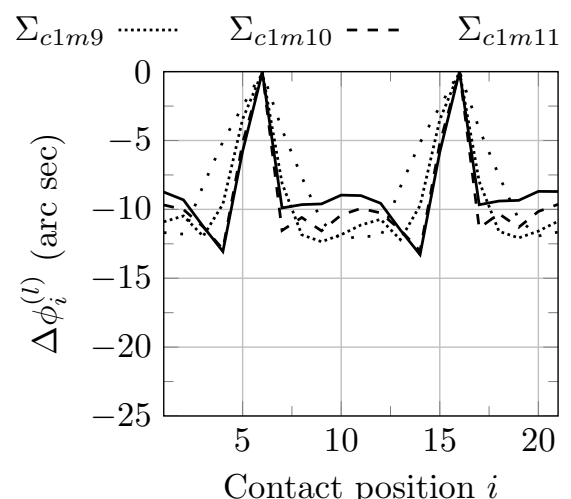

(b)

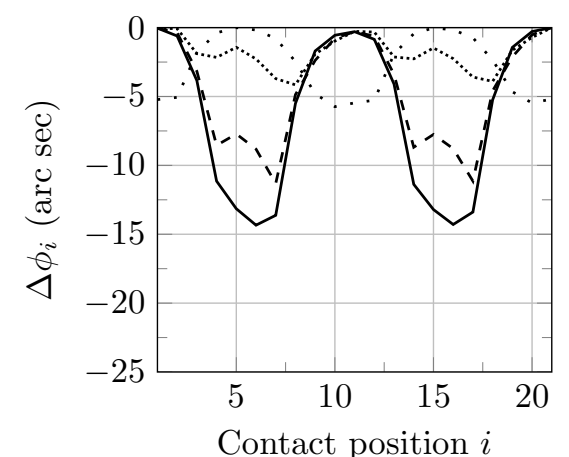

(c)

Fig. 13 Functions of (a) unloaded transmission errors, (b) loaded transmission errors, and (c) total transmission errors for geometries $\Sigma_{c 1 m 8}, \Sigma_{c 1 m 9}, \Sigma_{c 1 m 10}$, and $\Sigma_{c 1 m 11}$

ometry. Considering the modified compensated geometries, the profile crowning is even useful for elimination of some peaks on contact stresses due the rapid unload of teeth in contact. However the level of bending stresses is increased in modified compensated geometries respect to non-modified compensated geometries. The great advantage of modified compensated geometries is the reduction of the peak-to-peak transmission error (see Figs. 11, 12 or 13).

The evolution of the load intensity functions along the two cycles of meshing has also been investigated. Figure 15 shows the load intensity functions for all the 21 contact positions for geometries $\Sigma_{c 1}$ and $\Sigma_{c 1 m 2}$. It is observed that compensation of shaft deflections is actually working for each contact position.

\section{Conclusions and remarks}

The developed research allows the following conclusions to be drawn:
1. A procedure for compensation of shaft deflections in gear generation has been proposed and provides a uniform distribution of load intensity between gear tooth surfaces for the design load at the driving side.

2. Compensation of shaft deflections in gear generation is complemented with the predesign of different types of unloaded functions of transmission errors that allows the peak-to-peak value of total transmission error to be reduced.

3. The developed research has been accomplished along two cycles of meshing to assure that the uniform distribution of load between gear tooth surfaces is actually kept along the cycle of meshing, and that the evolution of contact and bending stresses is satisfactory for the proposed geometries.

The developed research has been focus on the compensation of shaft deflections for a given pinion rotation direction, which means that the non-driving side of pinion teeth is sacrificed. In fact, a finite element analysis of the gear drive considering the contact at the non- 


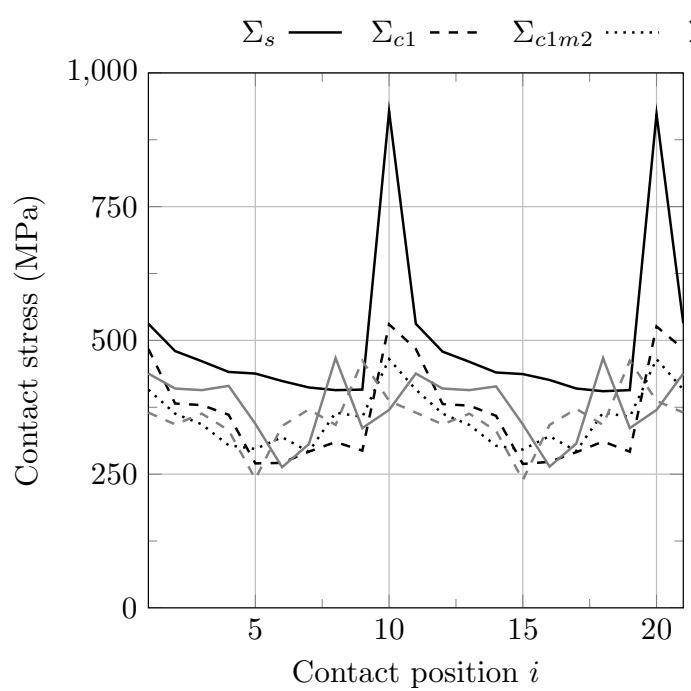

(a)

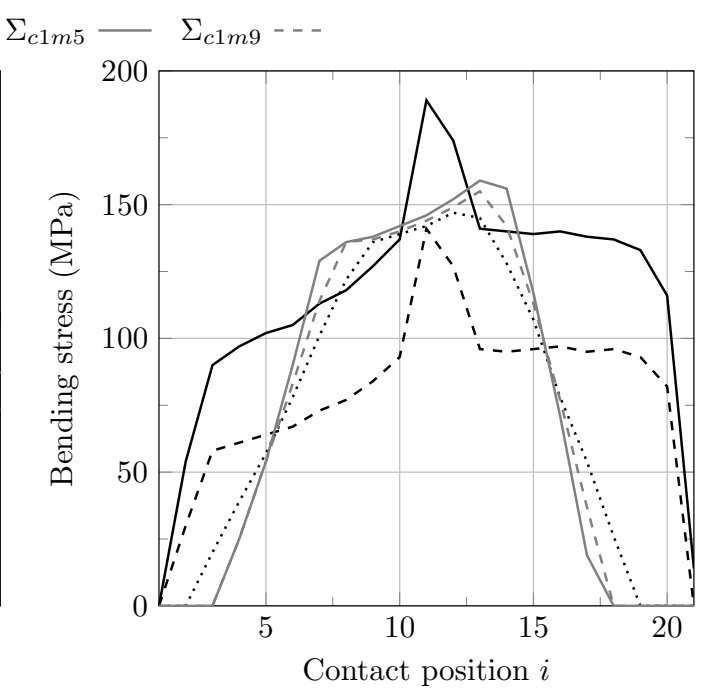

(b)

Fig. 14 Evolution of stresses for geometries $\Sigma_{s}, \Sigma_{c 1}$, and $\Sigma_{c 1 m 2}, \Sigma_{c 1 m 5}$, and $\Sigma_{c 1 m 9}$ : (a) contact Mises stresses, and (b) bending Mises stresses

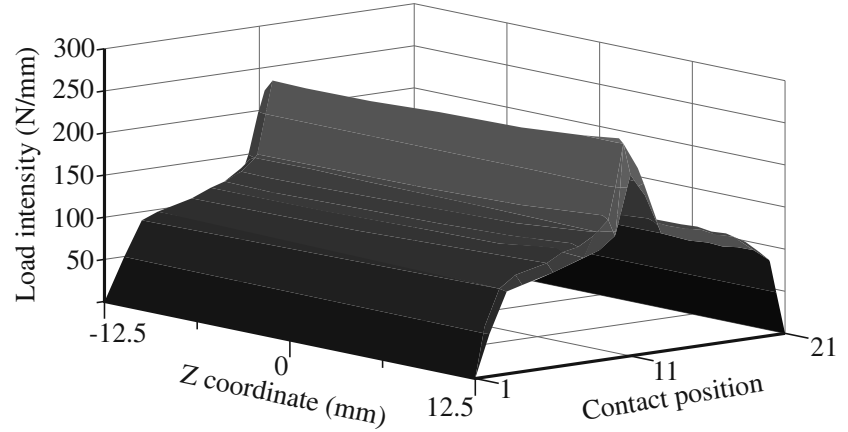

(a)

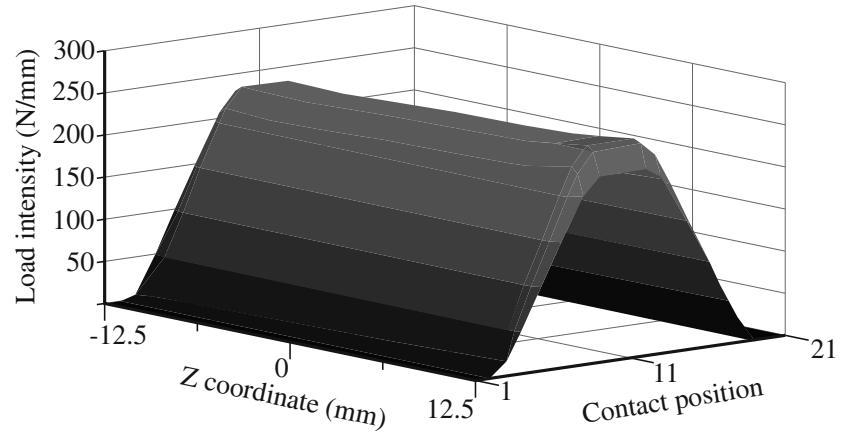

(b)

Fig. 15 Load intensity functions along the cycle of meshing for geometries: (a) $\Sigma_{c 1}$ and (b) $\Sigma_{c 1 m 2}$

driving side would provide an even worst load intensity function for the compensated geometries than for the standard geometry. Beside this, determination of backlash on the non-driving side is highly recommended for a better performance of the gear drive. All these topics are subject of future research.

Acknowledgements The authors express their deep gratitude to the Spanish Ministry of Economy and Competitiveness (MINECO) for the financial support of research project Ref. DPI2010-20388-C02-01 (financed jointly by FEDER) and DPI2010-20388-C02-02.

\section{References}

1. Harris SL (1958) Dynamic loads on the teeth of spur gears. Proc I Mech E 172(2):87-112
2. Gregory RW, Harris SL, Munro RG (1963) Dynamic behaviour of spur gears. Proc I Mech E 178(8):207-226

3. Optiz H (1968) Noise of gears. Phil Trans of the Royal Society 263:369-380

4. Bradley W (1973) How to design the noise out of gears. Machine Design 45(30):49

5. Welbourn DB (1979) Fundamental knowledge of gear noise - a survey. Proc Noise and Vibration of Engines and Transmissions, I Mech E, Cranfield, UK pp 9-14

6. Drago RJ (1980) How to design quiet transmissions. Machine Design pp 175-181

7. Wildhaber E (1962) Method and machine for producing crowned teeth. United States Patent Office, Patent No $3,046,844$

8. Novikov ML (1956) Ussr patent no. 109,750

9. Litvin FL, Lu J, Townsend DP, Howkins M (1999) Computerized simulation of meshing of 
conventional helical involute gears and modification of geometry. Mechanism and Machine Theory 34(1):123-147

10. Litvin FL, Fuentes A, Gonzalez-Perez I, Carnevali L, Kawasaki K, Handschuh RF (2003) Modified involute helical gears: Computerized design, simulation of meshing, and stress analysis. Computer Methods in Applied Mechanics and Engineering 192:3619-3655

11. Litvin FL, Gonzalez-Perez I, Fuentes A, Hayasaka K, Yukishima K (2005) Topology of modified surfaces of involute helical gears with line contact developed for improvement of bearing contact, reduction of transmission errors, and stress analysis. Mathematical and Computer Modelling 42(910):1063-1078

12. Gonzalez-Perez I, Roda-Casanova V, Fuentes A, Sanchez-Marin FT, Iserte JL (2012) A finite element model for consideration of the torsional effect on the bearing contact of gear drives. Journal of Mechanical Design 134(071007):1-8

13. Litvin FL, Fuentes A (2004) Gear Geometry and Applied Theory, 2nd edn. Cambridge University Press, New York (USA)

14. Sheveleva GI, Volkov AE, Medvedev VI (2007) Algorithms for analysis of meshing and contact of spiral bevel gears. Mechanism and Machine Theory 42(2):198-215

15. Fuentes A, Iserte JL, Gonzalez-Perez I, SanchezMarin FT (2011) Computerized design of advanced straight and skew bevel gears produced by precision forging. Computer Methods in Applied Mechanics and Engineering 200(29-32):2363-2377

16. Fuentes A, Ruiz-Orzaez R, Gonzalez-Perez I (2014) Computerized design, simulation of meshing, and finite element analysis of two types of geometry of curvilinear cylindrical gears. Computer Methods in Applied Mechanics and Engineering 272(0):321339 